\title{
ICT IN HEALTHCARE WITH HEALTH RECORD STANDARDS
}

\author{
Prashant Kanade \\ Research Scholar, Sir Padampat Singhania University, \\ Department of Computer Engineering \\ Udaipur, Rajasthan
}

\begin{abstract}
Details concerning a person's wellbeing, such as prescription history, immunizations, allergies, and medical test records, should be held in a uniform format. A systematic database of a person's health-care records will aid in assessing the appropriate treatment plan for someone who requires treatment at some point in their life. The majority of countries have their own health-carerecord-keeping scheme. The Ministry of Health and Family Welfare (Government of India) framed the guidelines for Electronic Health Records in our country (India) in 2013, and changes were made by April 2016. The need for the removal of the traditional health record system is stated in these guidelines. This paper's main goal is to propose an efficient model for an interoperable electronic health record system. The system is structured to keep track of the health records of patients in a systematic and user-friendly manner. Easy programmes have been written to convert stored data to communication standards such as HL7 and XML. The health-related details of a patient can be viewed and reused using HL7 and XML. There's even talk about getting specific data from the device. EHR systems in use in other countries are researched and used as a guide to develop an EHR system for India.
\end{abstract}

Keywords - EHR, EHR Standards, XML, HL7, Interoperability, EMR, Healthcare, ICD, SNOMED CT, Mapping of EHR Standards, ICT in Healthcare.

\section{INTRODUCTION}

Health care is subject matter of solicitation in every person's life. Every person will have interaction with medical practitioner since the birth. Vaccination, Treatment and lot many other health care related interactions are obvious for everyone. There are various standards such as ICD, SNOMEDCT, LOINC, UML to represent patient's disease information. HL7 and XML are one of the popular communication standards to share Patients information. These standards are beneficial to represent correct medical terms for patient data. Most of the doctors are using locally identified terms to represent patient health information[2]. Very few practitioners have adopted ICD to represent the patient disease information. In India there is a rare scenario to maintain patient's health care information in standard format and in computer memory in

\author{
Dr.Arun Kumar \\ Department of Computer Engineering \\ Professor, Sir Padampat Singhania University, \\ Udaipur, Rajasthan
}

electronic form. There are various challenges with reference to maintain health care information in the form of EHR [1]. In India the health care system has specific hierarchy from rural sector to urban sector. It is necessary to provide easy to use and systematic EHR system. EHR system should also assure meaningful use of healthcare information. In upcoming sections of this paper we have provided our findings on earlier EHR Systems, Indian scenario of Electronic Health Records, various EHR standards that can be adopted while following the guidelines provided by health authorities [5].

\section{REVIEW OF LITERATURE}

Amoussou J. (2010) has suggested in the article entitled Adventures in Computing mentioned that there should be perfect model to address the following issues with respect to Electronic Health Records. After modelling one can go for Requirements Gathering, The data modelling pattern adoption comes next. It is also necessary to map the model into HL7 Reference Information model termed as Continuity of Care Document (CCD) and Continuity of Care Records (CCR) that will help to facilitate the data exchange.

As discussed to design next generation EHR Data Models in the article published in Healthcare IT News. Shah (2012) has given guidelines to propose the model by involving the users of the system and other stakeholders of the system. The author has given few guidelines regarding the data model that should create patient centric system. Patient centric system can be able to provide access to all service providers. Priority is needed for Patient's information security and data should be preserved for life time.

Dr. David Blumenthal et al. (2012) specified that digital medical records help to maximize the quality of patient care. It helps to make accurate treatment related decisions as they can analyse the patient's medical history instantly. As all Laboratory tests or x-rays can be stored in the patient's electronic health record make it easier to track results. Alerts can be provided by system for specific disorders and allergies. E-prescribing allows doctors to send prescriptions instantly to the pharmacist; there is definite necessity to adopt electronic health records. It can be made available at reasonable cost and require an initial investment of time and money. Health service sectors that have implemented them have reported saving money in the long term. With the benefits that electronic health records promise, their widespread use has the potential to result in significant cost savings across our health care system. 


\section{International Journal of Engineering Applied Sciences and Technology, 2021 \\ Vol. 5, Issue 10, ISSN No. 2455-2143, Pages 175-185 \\ Published Online February 2021 in IJEAST (http://www.ijeast.com)}

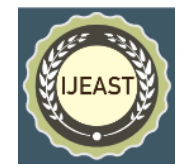

Though authors have mentioned advantages they have not mentioned any technical aspects regarding health record model or technical steps need to be adopted.

Maiedha Raza (2012) has given a detailed study of record keeping mechanism according to guidelines issued by general medical council, London. Authors have mentioned that standardised medical note keeping will result in good medical record keeping.

There is definite need of interoperability standards for health care information in India. People are now more aware about personal and family healthcare. Various health centres are providing their services to people. The health record information is stored in conventional manner that is in paper form. If the data is generated, stored based on industry standard, then it will provide the scope for quality in health care. There is a concept of meaningful use of data and adoption of health record standards will motivate to achieve the major intention of meaningful use of data. Indrajit Bhattacharya, et al. (2012) has represented need of interoperability standards. There are two major classifications of interoperability as i) Syntax: A required format that tells how data should be exchanged and ii) Services, it sees that how system understand data. The data that is shared or exchanged should be in appropriate format.

In a book entitled Electronic Health Records: Standards, Coding Systems, Frameworks and Infrastructures, authors have provided detailed information on health record coding standards as well as EHR systems. Authors are members of the National Electronic Health Authority. Standard Healthcare framework towards interoperability is discussed in this literature. This book is an overview of various healthcare standards. Sinha P et al. (2013) have mentioned the concept of continuity of care model. Continuity of care model is nothing but a methodology to record all interactions of the patient with each health centre department in real time following Figure represents the continuity of care model. As shown in the figure below it is clear that health record system should be capable enough to record all data beginning with patient's first point of contact i.e. registration and final interaction may be getting the medicines from medicine delivery unit or pharmacy.

When patient record is shared to third party stake holders even if they are authorised users it is necessary to preserve these records with high level of security and encrypted before sharing. Omole G.A. et al.(2016) have represented privacy preserving techniques. If records are available online they can be accessed anywhere and anytime. Each patient can be uniquely identified either by a serial number or by a unique identification number provide by government authorities.

Mandatory Access Control (MAC), Discretionary Access Control (DAC) and Role Based Access Control (RBAC) can also be incorporated if records are preserved on a central server or the cloud-based environment. Easy to use system can be developed for small hospitals and Clinics using web based cloud environment. We can implement all security or access control mechanism easily if we have direct access to system dashboard. Cost effective system is also on the cards if we have dedicated server environment.

Government of India has given a thought to set up a statutory body called National Electronic Health Authority (NeHA). In a small section it is mentioned that Patients in India are facing various difficulties. Many public and private hospitals have built their own healthcare systems or hospital information systems over time that have served patients well, but without an emphasis on standards implementation, interoperability, or system interconnection through hospital environments that can lead to quality of care - resulting in inadequate outcomes. Such non-interoperable discrete islands of knowledge have posed significant barriers to successful information sharing among healthcare participants, a problem exacerbated by India's multiple health service boundaries and geographic distances.

To propose some model with respect to EHR system detailed study is carried out of various EHR systems whose summary is provided in Annexure 1 after references. Sweden has a nationwide health record framework and same is suitable for India as we have huge population and it will help in providing remarkable ease of meaningful use of EHR.

\section{History OF MEDICAL ReCORDS}

Early efforts to adopt Electronic Health records took place in early 1960s and 70s. The earlier health information systems were named as clinical information systems. In 1968, the Computer Stored Ambulatory Record (COSTAR) began at Massachusetts General Hospital. Developed in collaboration with Harvard, COSTAR included some novel features. The COSTAR was separated into parts as the accounting portions which was totally isolated from healthcare related information and the other part that consists of clinical information. The clinical part consists of extraneous information that increases efficiency. The system also had a flexible vocabulary; its database recognized multiple terms for the same disease. The biggest advantage of the system was that it facilitated users to recognize a given condition across the health system despite variations in terminology at different institutions. Means it was able to identify the description of disease recorded in other systems with different terms.

Since the 1980s, more concerted efforts have been made to increase use of EHR. The Institute of Medicine (IOM) recognized the need for serious analysis of paper health records and relevant work was carried out till 1997. They provided one of seven key recommendations for improving patient records, and to propose a means of converting paper to electronic records. Computer-Based Patient Record Institute (CPRI) was established by group of various industries. CPRI helped to break down barriers to EMR development. CPRI is 


\section{International Journal of Engineering Applied Sciences and Technology, 2021 \\ Vol. 5, Issue 10, ISSN No. 2455-2143, Pages 175-185 \\ Published Online February 2021 in IJEAST (http://www.ijeast.com)}

now merged with the Health Information and Management Systems Society, HIMSS.

\section{CHALLENGES IN INDIA}

In 2013, Ministry of Health and Family Welfare notified Electronic Health Records (EHR) Standards for India. The set of Standards given therein were selected from successful standards applicable to EHRs from around the world. Detailed analysis is carried out about suitability and applicability of these standards in India by some expert group. Ministry of Health and Family Welfare (e-governance Division) issued a notice regarding EHR in 2016. Now these Standards have been improvised and made according to the ever changing need of the mass.

Ministry has also specified various standards which are already taken into consideration and some additional standards that need to be incorporated. In these guidelines detailed recommendation on interoperability standards and clinical informatics standards, data ownership, privacy and security aspects are discussed.

Following are the salient features of the guidelines given by Govt of India Ministry of Health and family welfare:

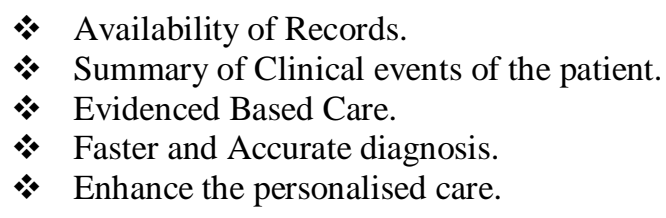

The number of physicians in India are very less (10 Lakhs for $133 \mathrm{Cr}$ population). Among these 10 Lakh medical practitioners only 1.5Lakh Doctors in Public healthcare to serve patients. There is absolute non existence of patient centric care in our Country. As discussed already there is a huge shortfall of access to healthcare services in our country.

All private hospitals and medical facility centres have their own computer system but it is limited to administration purpose. Clinical documents are not digitised in most of the health centres.

In India there are very less medical facility units to provide healthcare to huge population. Below mentioned graph specifies the status of medical centres in India. We can observe from the below mentioned table that, there is a growth in number of health centres in India, but these numbers are much below the requirement as far as population of India (133 $\mathrm{Cr}$ ) is concerned.
Table 1: Statistics of Number of Govt Health Centres

\begin{tabular}{|c|c|c|c|c|c|}
\hline YEAR $\rightarrow$ & 2005 & 2012 & 2017 & 2018 & 2019 \\
\hline Sub Centre & 146026 & 148366 & 156231 & 158417 & 160713 \\
\hline $\begin{array}{c}\text { Primary } \\
\begin{array}{c}\text { Health } \\
\text { Centres } \\
\text { (PHCs) }\end{array}\end{array}$ & 23236 & 24049 & 28863 & 25743 & 30045 \\
\hline $\begin{array}{c}\text { Community } \\
\text { Health } \\
\text { Centres } \\
\text { (CHCs) }\end{array}$ & 3346 & 4833 & 5624 & 5624 & 5685 \\
\hline
\end{tabular}

Health centres in India; Data Source: National Health Mission India Website (Govt. of India).

There are some barriers, such as a shortage of personnel, infrastructure, and awareness among all service providers about the importance of properly documenting health care data.

Allopathy, Homeopathy, Ayurveda, and Unani are some of the treatment methods used in India. There are also a number of successful treatment approaches that have been shown to be effective for a number of diseases. In urban and semi-urban areas, the majority of people depend on general practitioners and conventional care methods. All treatment-related history must be reported in a standard format, and this will certainly help India's healthcare services. Health services are available in India through a number of health facilities, including Primary Health Centres, Community Health Centers, and Sub Centers.

General practitioners provide treatment through personal pharmacies, government polyclinics, and private polyclinics in each field, in addition to the aforementioned Health Care System. Under the 'DISHA' act, patients whose lives have ended in hospitals during treatment or who have Medico-Legal cases have their records kept for life. The majority of general practitioners do not keep track of their patients' information. There have been some good examples of correctly framed treatment. The record cannot be made available for exceptional analysis because it is not held in the specified format.

To make it varied aim EHR is important in India. It can be made available for different users for various purposes. It can also be made open to all stakeholders, both direct and indirect. Here are a few benefits of using an EHR system:

- Immediate access to health-related data.

- Long-term stability

- Reliability

- The opportunity to work together

- Convenience

It is also important to understand the following points in order to arrive at the above-mentioned beneficial features, such as 1) 
Patient Centric Health Information System. 2) The userfriendliness of the product. 3) Interoperability and portability must be discussed. 4) Discussing questions of protection and privacy. 5) Ownership, governance, and traditional issues that must be considered.

\section{EHR STANDARDS}

In this section we will have a brief summary of various EHR standards recommended by Ministry of Health and Family welfare [6]. In India most of the medical practitioners are using technical terms for diseases or locally identified terms while recording the diagnosis, if they adopt standard terms it will be feasible to share and analyse healthcare data.

\section{A. Identification Standards:}

UIDAI is nothing but Unique identification authority of India and they have provided the unique identity of a person based on his demographic and biological credentials, it is termed as Aadhaar Number and same can be used as personal identification number. This is preferred for a patient's identification. If person do not have any UIDAI registration then any registration provided by Local Authorities can be used or if person is employed in government any central or State Government issued Photo Identity number can be used.

\section{B. Healthcare record standards}

Various health care standards are recommended by National Resources Centre, Pune. They are as mentioned here.

1) ICD. 2) SNOMED CT.3) LOINC. 4) DICOM. 5) UML and communication standards as HL7 and XML. Apart from these standards it is also necessary to store patient's Demographic information including a unique identifier is necessary in a health record system in order to capture identifying information as well as identifiers for linking other medical artefacts logically as well as physically [14]. A health record system must have provision to include patient identifiers of following types.

\section{B Diseases Classification standards}

SNOMED CT: Systemised Nomenclature of Medicine

\section{Clinical Terms}

SNOMED CT is now well known acronym. SNOMED CT is owned, maintained and distributed by SNOMED International, United Kingdom. It is comprehensive, multilingual clinical healthcare terminology in the world. It contains 443,237 concepts, almost 1.3 million descriptions and nearly two and a half million relationships.

Salient Features of SNOMED CT

- Is the most comprehensive, multilingual clinical healthcare terminology in the world

- Is a resource with comprehensive, scientifically validated clinical content
- Enables consistent, process able representation of clinical content in electronic health records

- Is mapped to other international standards

- Is already used in more than fifty countries

SNOMED CT content is represented using three types of component:

- Concepts

- Descriptions

- Relationships

SNOMED CT is represented by a decimal code having 8 to 10 digits. Let us consider an Example: 22298006 which is code for Myocardial Infarction (disorder) and Further this can be represented as follows:

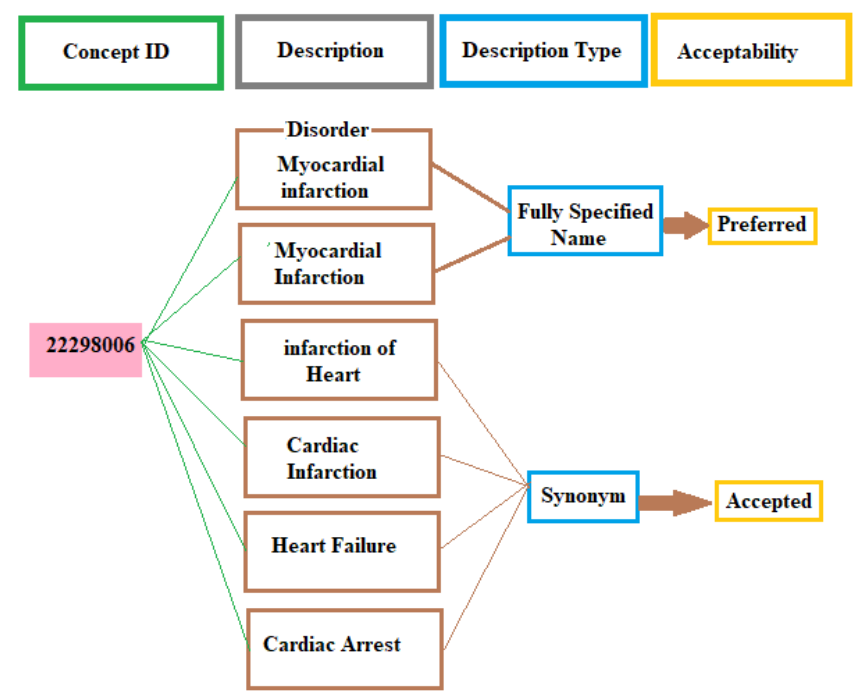

Figure 1: SNOMED CT Representation for Myocardial infraction

Snomed CT standard has major components as a concept id, Description, Description type and accessibility. As shown in the figure above the 8 digit number is called as concept id, then in the description fully specified name and it is synonym will be represented. If required the type of description will be specified whether it is Synonym or Fully Specified name. In the acceptability the preferred and acceptable terms will be labelled.

\section{ICD: International Classification of Diseases:}

ICD is nothing but International Classification of Diseases.ICD is statistical Classification System which is used to monitor the incidence and prevalence of diseases and other Health problems. It contains codes for diseases, signs and symptoms, abnormal findings, complaints, social circumstances, and external causes of injury or diseases. Major Users of ICD are Clinicians and other Health service providers. ICD is owned by World Health Organization.

ICD is a mono-hierarchy and it is structured as shown in the figure below. The main concept of ICD is that it Start with 
alphabets A B C mapped with Numbers as shown in the example below for diseases of respiratory system.

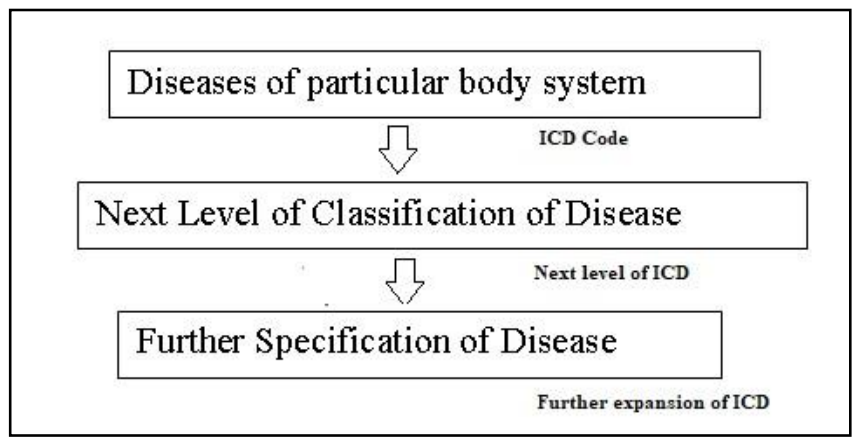

Figure 2: ICD Mono-hierarchy representation

DICOM: DICOM is nothing but Digital Imaging and Communications in medicine this is a standard for communication and management of medical imaging information and related Data.DICOM was developed to Promote communication of digital image information .It facilitates Picture archiving and communication systems that can interface with other hospital systems and to create diagnostic information.

LOINC: LOINC is Logical Observation Identifiers Names and Codes, LOINC is a set of identifiers, names and codes for identifying health measurements, observations and documents. It is universal code system will help to receive and send results from their areas for comparison and consultation.

UML: Unified Medical Language was Initiated by National Library of Medicine to support ICT in Health Care. It has following major Blocks as 1. Meta-thesaurus 2. Semantic Network 3. Specialist Lexicon and Lexical Tools

Meta-thesaurus concept has a) Concept Unique Identifier b) Lexical Unique Identifier c) String unique Identifier. d)Atom Unique Identifier. Following figure shows detailed description of UML representation of a disease.

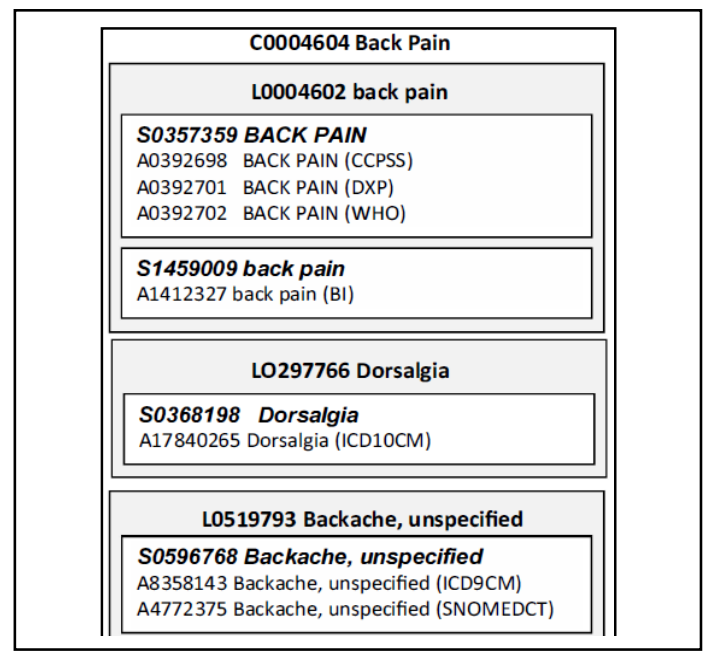

Figure 3: UML Standard Representation

\section{Communication Standards:}

Health Level 7 (HL7): This refers to a set of international standards for transfer of clinical and administrative data between software applications used by various healthcare providers. These standards focus on the application layer, which is "layer 7" in the OSI model.

HL7 message can be easily generated by using HL7 parser or most of the programming languages have API for HL7. Below is the typical HL7 message and its description.

Whenever HL7 message is created it is added with message header along with patient's personal information and medical data for a patient. This message can be retrieved back to get the textual format or human readable format of the information of a patient. Figure 8 shows the regenerated message

$$
\begin{aligned}
& \text { MSH|^ }\left.\right|^{\wedge} \& \mid \text { HL7Soup|Instance1|HL7Soup|Instance2|2009110210 } \\
& \text { 22||MDM^^T01^MDM_T01|64322|P|2.5.1 } \\
& \text { EVN|T01|200911041022|200911051022|1|743789 } \\
& \text { PID|1|103456|103456||Kanade^ }{\text { Prashant }{ }^{\wedge} \wedge|| 19741122|M||| E 14} \\
& \text { Satguru Apartment } \\
& \text { ^^Thane^^400601^IN|||||M|HIN||46264212||||Thane|N|| } \\
& \text { PV1|1|I|CARE }
\end{aligned}
$$

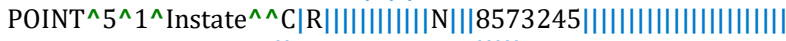

$$
\begin{aligned}
& \text { ||200911011122||||| } \\
& \text { TXA|1|CN|RTF|||||20081127100756||||24567^FACIL||||34252.rtf } \\
& \text { |DO|R|AV|AC||| }
\end{aligned}
$$

Figure 4: HL 7 Message Description

As specified in the figure above message header is specified as MSH that contains information of tool that is used along with its instance. Patient first visit to medical centre is termed as EVN patient's identification as pid and transactions as TXA.

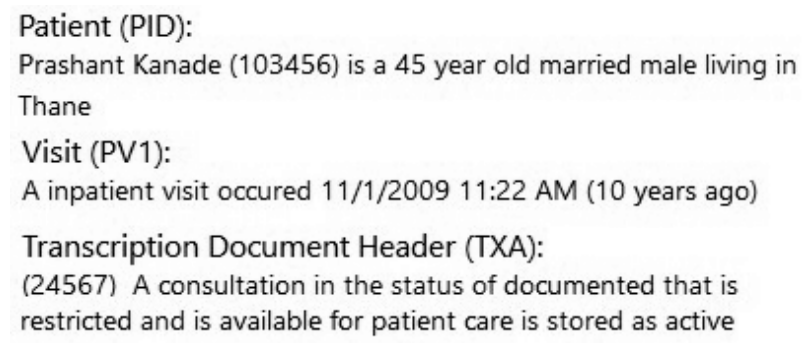

Figure 5: HL7 Description.

XML: $\mathrm{Xml}$ is also found very successful communication standard. Major advantage of XML isthat it is easy to implement and easy to share communication standard. The XML standard is a flexible way to create information formats and electronically share structured data via the public Internet, as well as via corporate networks. XML is found 


\section{International Journal of Engineering Applied Sciences and Technology, 2021 \\ Vol. 5, Issue 10, ISSN No. 2455-2143, Pages 175-185 \\ Published Online February 2021 in IJEAST (http://www.ijeast.com)}

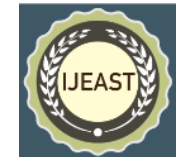

suitable for handling data with a complex structure or atypical data. Data can be described using mark-up language. XML is also has human- and computer-friendly format. Another advantage of XML is that it handles data in a tree structure having one-and only one-root element. XML is excellent for long-term data storage and data reusability. Simple programs can be written to convert the content in XML and also can be easily parsed in required Format. In this paper some sample output are represented by converting patient data into XML Format.

\section{DESIGN CONSIDERATIONS OF EHR SYSTEM}

Before having a detailed design description of EHR System it is necessary to highlight some points pertaining to need of Standards in EHR System. Whenever there is a need to share patient's medical record to other medical expert or other health care centre it is necessary that it should be provided in standard format recommended by the national health authority [13]. If medical records are in scattered format it is very difficult to analyse the record and that may extend the delay in decision making about the treatment. Standard terms will help in meaningful use of healthcare information.

Web based system is recommended with virtual private server hosting. A VPS, consists of a virtual operating system that is provided on the server and provides dedicated (private) resources to other virtual servers. The dedicated VPS hosting environment is created on a host machine. VPS hosting is generally lower in cost than dedicated hosting but provides better reliability, security, and performance than shared hosting. When a VPS Hosting is made available we can install applications and make customised web pages irrespective of hosting provider.

VPS is often a great option for Software as a service and it also provides secure and affordable backup environment. Few key benefits to VPS hosting are nothing but

- Reliability in performance

- Value

- Control

- Customization

- Growth

- Data protection

- Security

Cpanel hosting is very popular webhosting control panel. It provides a well managed user interface and various tools to configure the web pages. The file manager of cpanel simplifies the process of hosting a web site. It has facility to administer web content using a three-tier structure.

To begin with implementation of EHR it is recommended that a secure system with sufficient web space is required to adhere to this we have a web space with webhost management system and a dedicated domain name as ehrsystem.co.in and it is configured to host all modules that are recommended. As per the systematic study we recommend a system with two major parts as recording healthcare information and converting the health care information in HL7 or in XML format.

Web based system is easy to configure and easy to use system. This can be a conventional system to implement EHR based system. Various Human Machine Interaction styles are adopted in web based system[3]. The major disadvantage of the web based system is high demand of security arrangements as it has its own limitations. To initiate the EHR system Web Based system is recommended. In this initial approach PHP MY SQL and JASON is widely adopted.

We have created simple HTML programs and PHP scripts to provide simple but systematic healthcare system. As this is the primary approach See Figure 9 below. We have not provided any intelligent system for data recording but secured approach is proposed to access and use healthcare data. In earlier few publications we have proposed a system for General practitioners and same can be further stored in standard format on centralised nationwide accessible system. The typical user interface is showcased in Annexure.

As shown in the figure 6 below we have a typical EHR System that will have salient features recommended by ministry of health and family welfare (MOHFW) govt. of India. A system is motivated from the EHR system initiated by Sweedons NPO having a one Ptient One record Policy. Major focus is on EHR System that will have salient features recommended by ministry of health and family welfare (MOHFW) govt. of India.

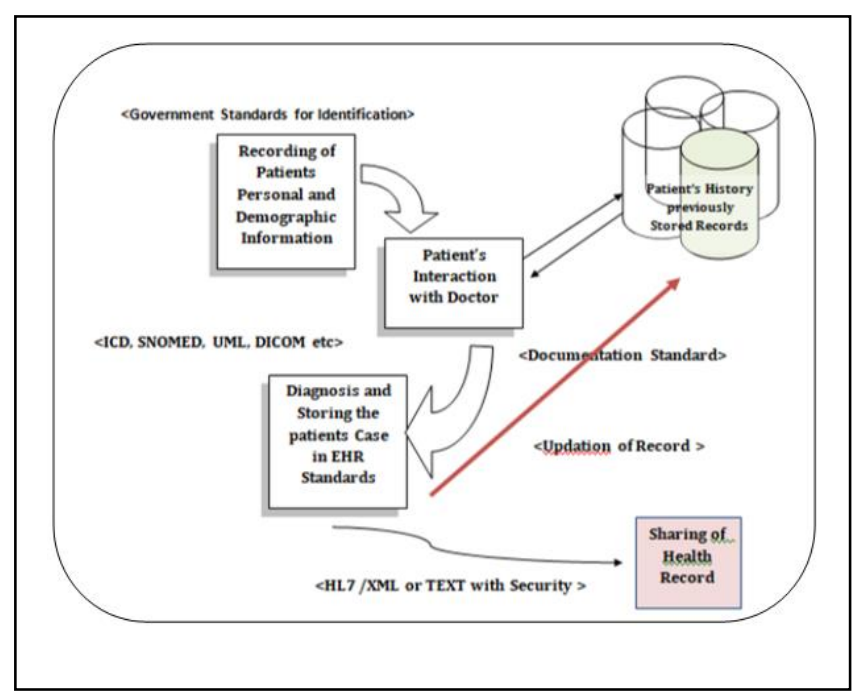

Figure 6: Generalised Block Diagram of intended system

\section{MAPPING OF STANDARDS}

Normally Doctors use FSN i.e. fully Specified Name or Locally Identified name to record patient case. It is feasible for them to store record in less available time. We can provide them a facility on a digital device to store the information in prescribed standard. Here in this system the efforts are made 


\section{International Journal of Engineering Applied Sciences and Technology, 2021 \\ Vol. 5, Issue 10, ISSN No. 2455-2143, Pages 175-185 \\ Published Online February 2021 in IJEAST (http://www.ijeast.com)}

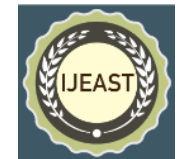

to store the information in two standard formats for one FSN or Local Name of the disease. That mapping will help to store disease information in ICD as well as in SNOMED code for each medicinal interaction.

It is very difficult to instantly find the equivalent standardised name for the fully specified name. Each authority that has developed these standards have their browsers or directory to identify the equivalent identification of the medical term. If the mapping table is generated by including all necessary standards; one can definitely get benefit of using these standards. Following Table No 3 is a compact version of the mapping table.

To create mapping of these standards special efforts need to be taken to locate the correct equivalent name and verify it be Subject Matter Experts. It is mandatory to involve experts in this mapping process.

Table 2 : Mapping of EHR Standards

\begin{tabular}{|c|c|c|c|}
\hline Fully Specified Name & ICD & UMLS & SNOMED CT \\
\hline $\begin{array}{c}\text { Cholera due to Vibrio } \\
\text { cholerae } 01 \\
\text { biovarcholerae }\end{array}$ & A000 & C0494021 & 63650001 \\
\hline $\begin{array}{c}\text { Cholera due to Vibrio } \\
\text { cholerae } 01 \\
\text { biovareltor }\end{array}$ & A001 & C0343372 & 63650001 \\
\hline Cholera, unspecified & A009 & C0008354 & 63650001 \\
\hline Typhoid & A0100 & C0041466 & 4834000 \\
\hline $\begin{array}{l}\text { Typhoid fever, } \\
\text { unspecified }\end{array}$ & A0100 & C0041466 & 772154007 \\
\hline Typhoid meningitis & A0101 & C2880086 & 192648008 \\
\hline $\begin{array}{l}\text { Typhoid fever with } \\
\text { heart involvement }\end{array}$ & A0102 & C2880088 & $\begin{array}{c}108479100011910 \\
0\end{array}$ \\
\hline Typhoid pneumonia & A0103 & C0339956 & 45312009 \\
\hline Typhoid arthritis & A0104 & C2880089 & 4834000 \\
\hline $\begin{array}{c}\text { Typhoid } \\
\text { osteomyelitis }\end{array}$ & A0105 & C2880090 & 483400060168000 \\
\hline $\begin{array}{l}\text { Typhoid fever with } \\
\text { other complications }\end{array}$ & A0109 & C2880091 & 4834000 \\
\hline Paratyphoid fever A & A011 & C0343375 & 76623002 \\
\hline Paratyphoid fever B & $\mathrm{A} 012$ & C0343376 & 71085009 \\
\hline Paratyphoid fever C & A013 & C0343377 & 51254007 \\
\hline $\begin{array}{c}\text { Paratyphoid fever, } \\
\text { unspecified }\end{array}$ & A014 & C4750347 & 85904008 \\
\hline Paratyphoid suspect & A014 & & 772164003 \\
\hline Salmonella enteritis & $\mathrm{A} 020$ & $\mathrm{C} 0275783$ & 397503006 \\
\hline Salmonella sepsis & A021 & C3163971 & 449083008 \\
\hline $\begin{array}{l}\text { Localized salmonella } \\
\text { infection, } \\
\text { unspecified }\end{array}$ & A0220 & C0152487 & 47375003 \\
\hline
\end{tabular}

Any user who is going to store the patient case record will simply select the Local name or FSN for the disease and within fraction the corresponding information will be stored in the database table. We can also retrieve the same in required format for particular Patient based on simple web based programs for the same.

As discussed in previous section regarding EHR standards, web programming techniques are applied to record patient's health care information [9]. Disease information is maintained in a database with respect to schema specified in the diagram below. The same schema is adopted while recording patient's healthcare information. Practitioners and data entry operators will only insert some key terms about the disease most probable medical terms for the disease or diagnosis and system will use the mapping information from the database and record will be generated. This readily available mapping of disease information with EHR standards will assure the standardised healthcare information of the patient and same can be used when and where required. Annexure Figure 2 has complete representation for the same.

We can access healthcare related information by implementing SQL query and a user interface. In this system we have also applied Natural Language processing method of SQL and that will also help to access patient information based on some key terms. We can browse the patients with specific disease based ob FSN, SNOMEDCT or UML key terms. Next section has detailed discussion and few set of results.

\section{ACCESSING HEALTH DATA}

Accessing Health data will be especially for researchers who wish to apply some analytics to the health data. It will be also useful for organizations to take any strategic decision from the available systematic data[4]. We can also retrieve information about specific patient or for specific stakeholder in the health care system. To make the access simpler; natural language processing can also be applied[7].

We can access specific record from the system using sql query and that is suitable for users who are comfortable with the ICT techniques. We can use patient identification number (Normally termed as Patione ID) to access record. Same is mentioned in Following figure 7.

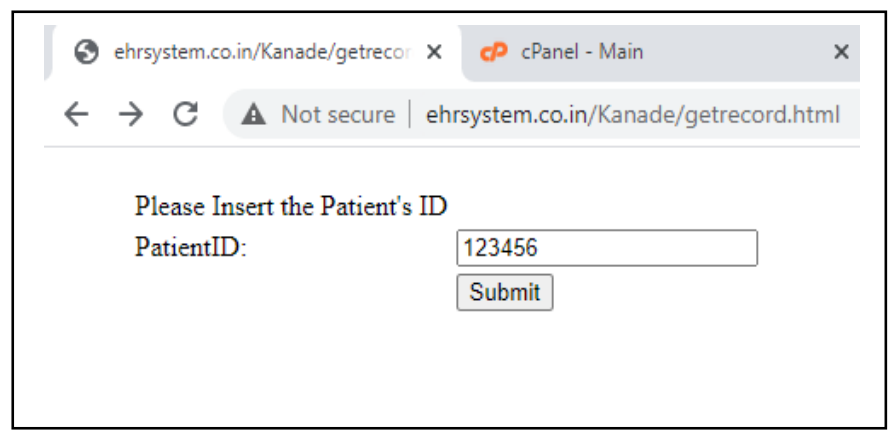

Figure 7: Searching Record with Patient ID 
We know that, users can be provided with facility to type their query in simple English sentence as well as we can get their speech converted into text. Further that text can be used as an input string to access relevant data.

In MySql there is a special method termed a s NLP FULL TEXT Search. In NLP Full Text Search technique MySql will look for rows or documents that are relevant to the freetext natural human language query, for example user types the English sentence as "I want record of patients suffering from Fever" then we can use NLP text based search mechanism of MYSQL to get resultant list of all patients registered in the system and they are suffering from fever. Please see the figure number 12 below that is a snapshot of the module implemented on our EHR System portal http://www.ehrsystem.co.in.

Figure 8(a) and Figure 8(b) is the result of NLP Full text Search technique and that is found very effective to search records with either FSN or ICD or SNOMED.

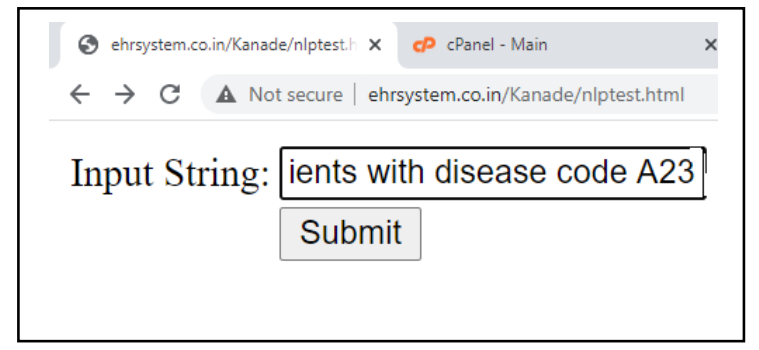

Figure 8 (a) NLP Search Technique (Input)

\begin{tabular}{|c|c|}
\hline$\leftarrow \rightarrow C$ & A Not secure | ehrsystem.co.in/Kanac \\
\hline \multirow[t]{7}{*}{ INPUT STRING } & I want patients with disease code $\mathrm{A} 23$ \\
\hline & 789023456 licver $A 234856678978$ Kalc \\
\hline & 8969123456 lever $A 234856678978$ joshi \\
\hline & $9800123456 \mathrm{~J} \mathrm{Cver} \wedge 234856678978$ ghulc \\
\hline & 9991123456 lever A23 4856678978 Dr.Jigar \\
\hline & 444423456 lever A23 4856678978 Joshi \\
\hline & $2.5450 \mathrm{l} 23456 \mathrm{l}$ ever $\wedge 2.348560 / 39 / 8$ shah \\
\hline
\end{tabular}

Figure 8(b) Result of NLP Full Text Search

\section{COMMUNICATION STANDARD XML AND HL7}

A) XML representation: We can use XML to share patient's information between health service provider and hospitals. $\mathrm{XML}$ is used as the standard language of communication in the context of Web Services. It works regardless of programming language or operating system used in the implementation of applications. Following is the typical XML representation for a patient record which is available in a database implemented on web portal http://www.ehrsystem.co.in. Same record can be reused by converting it into a table form. XML is lightweight and hence can be transmitted irrespective of any communication channel.

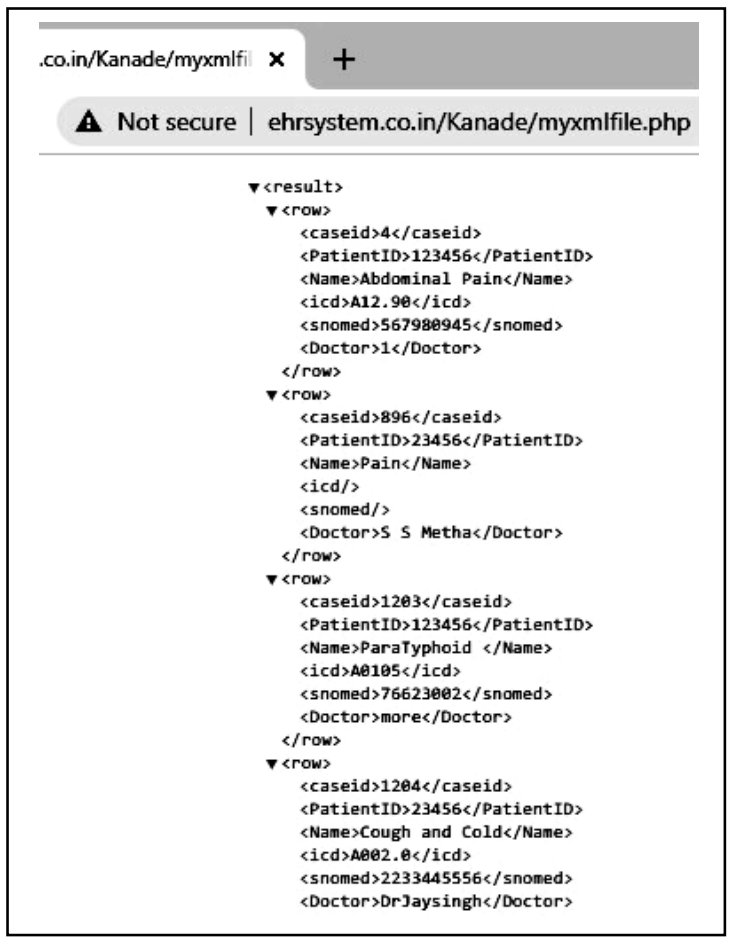

Figure 9 : XML Representation of the Health Data

(Ready to reuse)

B) HL 7 Communication Standards

HL7 Can support exchange of Healthcare Data across various Health Care applications, supporting different Communication environments.HL7 can provide representation for patient administration,

billing, reports, record management and other necessary information

Messages in HL7 are of two Types:

1. Event: Real World events happening in Healthcare that comprises of transfer of health information. Basically used for Notifications and can be identified by a field called MSH

2. Query: This messages are used to query health care information of one or more patients. There are various response messages that contain required meaningful Information.

Every HL7 message will comprise of following fields as

- MSH: Header segment of HL7 Message and it comprises of source and destination application name, message type, message country and other technical Data

- PID: Patients Identification and demographic information, Name, Race, Gender, Status of Family, Phone etc. 


\section{International Journal of Engineering Applied Sciences and Technology, 2021 \\ Vol. 5, Issue 10, ISSN No. 2455-2143, Pages 175-185 \\ Published Online February 2021 in IJEAST (http://www.ijeast.com)}

- PV1: Patient visit Segment, information related hospital visit, Doctor attending, cause etc.

- OBR: Observation Request for any diagnosis

- OBX: Observation Results

To record all above information and convert in HL7 format a special python code is written and python API is used to convert the patient data in HL7 format. Following figure shows the corresponding user interface and HL7 output of the record. The HL7 parser can be used to convert the record back to actual record that can be easily reused and analysed.

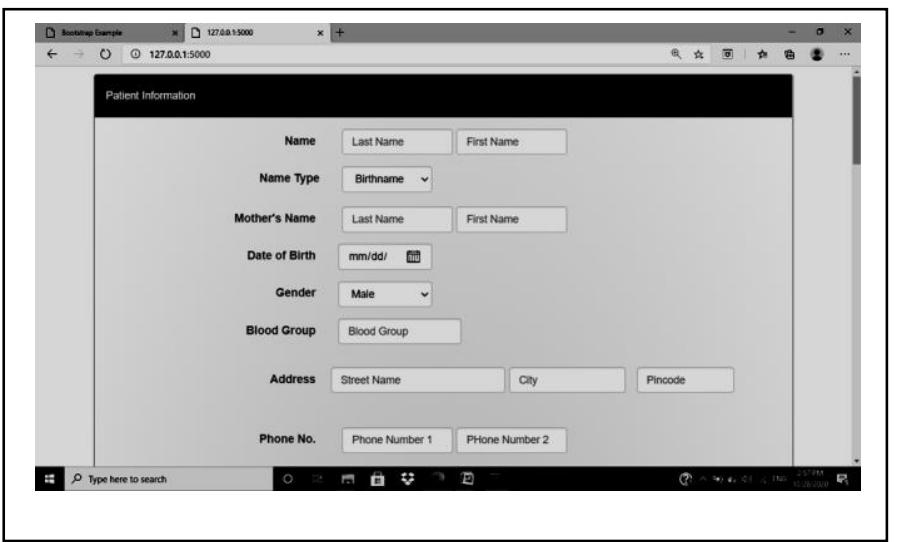

Figure 10: GUI to gather patient data to be converted in Python

After submitting the record system allows us to convert the record into HL\& and same can be parsed into typical document using various HL7 parsers. See figure 17 below.

System will return Typical HL7 message as follows

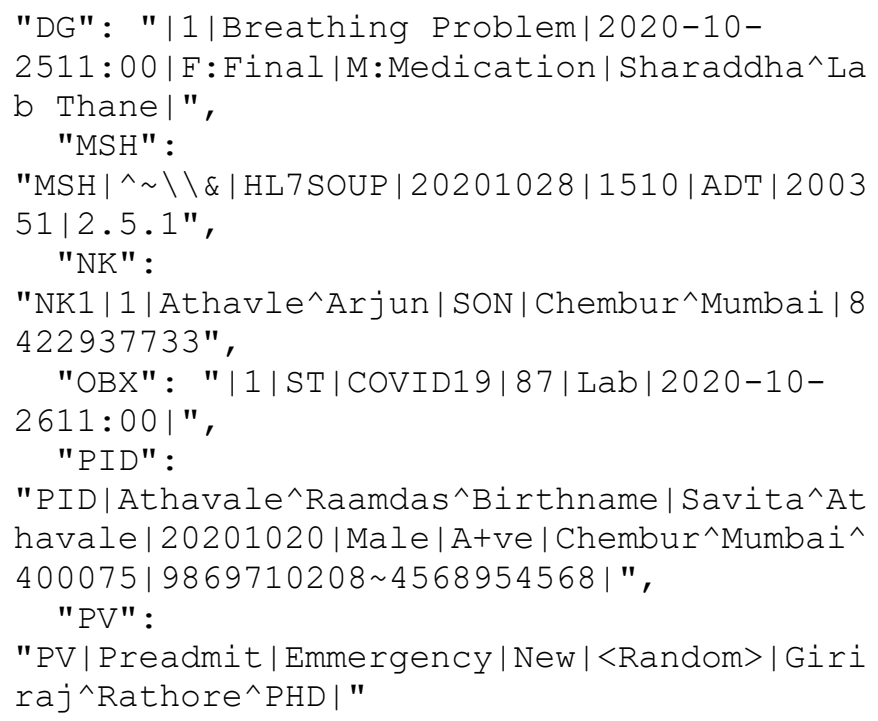

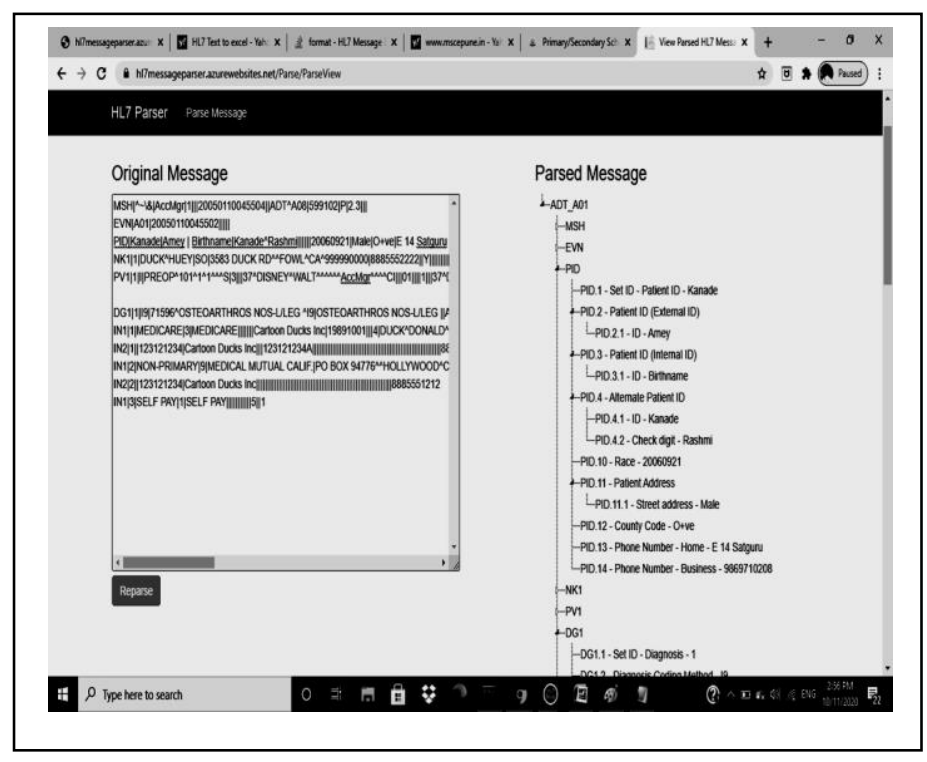

Figure 11: HL7 Parser and its usage to convert HL7 Message into Text format

Hence we can further make necessary changes as per the requirement in our program and can provide a very effective EHR system.

\section{CONCLUSION}

With this we can conclude that maintaining patient record in digital format initiated way back in 1960 but it was the then in very initial phase. Most of the countries have also initiated Electronic Health Record Systems. India has also issued guidelines for EHR. National electronic Health Authority has also prescribed standards to record health data and a simple easy to use and effective EHR system can be developed.

There are certain challenges that are identified towards the interoperability and these challenges are with respect to:

1. Exchange of Information Between two Different Healthcare Systems

2. Information model with EHR Standards recommended by Govt of India

3. Digital Healthcare Model or Digital System Integration.

Other Challenges are in

1. Making Doctors Confident Computer Users.

2. Providing better System with less Human Efforts

3. Automation to record Health Information with EHR Standards

Our major aim is to provide interoperability to health related information and patient's health related information can have meaningful use of data. As discussed already XML and HL7 are communication standards and that can be used for sharing patient's health related information. Health record can be parsed into XML or in HL7 using various programming techniques. 


\section{REFERENCES}

1. Bhartiya S., Mehrotra D., Girdhar A. Issues in Achieving Complete Interoperability while Sharing Electronic Health Records, International Conference on Information Security \& Privacy (ICISP2015), 11-12 December 2015, Nagpur, INDIA , (2016) Procedia Computer Science, 78 , pp. 192-198.

2. Bhattacharya I and Muju S (2012), Need for Interoperability standards for Healthcare in India, CSI Journal of Computing, VOL 1. No.4,2012

3. Calvanese D, Giacomo G De, Domenico L, Data Management in Peer to peer Data Integration Systems uploaded on https://www.researchgate.net/profile/Diego_Calv anese

4. David W. The Road to Implementation of Electronic Health Record, Baylor University Medical Center Proceedings 19:4,311-312, DOI: 10.1080/08998280.2006.11928189

5. EHR Standards for India(1), Guidelines given by Ministry of Health and Family welfare,April2016, e-governance Division

6. EHR Standards for India(2), standards at a glance on a portal of National Resource Centre for EHR Standards shared on https://www.nrces.in/standards-forIndia\#tstandards at a glance page 1-8

7. Jian W.-S., Wen H.-C., Scholl J., Shabbir S.A., Lee P., Hsu C.-Y., Li Y.-C. The Taiwanese method for providing patients data from multiple hospital EHR systems (2011) Journal of Biomedical Informatics, 44 (2) , pp. 326-332.

8. Kuo T, Kim H, Ohno-Machado L. Blockchain distributed ledger technologies for biomedical and health care applications. J Am Med Inform Assoc 2017 Nov 01;24(6):1211-1220. [doi: 10.1093/jamia/ocx068] [Medline: 29016974]

9. Mantri M, Gaur S,Sinha P (2010), "Model and Process Interoperability between Clinical Standards", Conference: 6th International Conference of Telemedicine Society of India (Telemedicon'10) At: Bhubaneswar, Odisha, https://doi.org/10.13140/2.1.1390.8808

10. Meinert E, Alturkistani A, Foley KA, Osama T, Car J, Majeed A, Van Velthoven M, Wells G,
Brindley D Blockchain Implementation in Health Care: Protocol for a Systematic Review, JMIR Res Protoc 2019;8(2):e10994 , URL: https://www.researchprotocols.org/2019/2/e1099 4 DOI: 10.2196/10994, PMID: 30735146, PMCID: 6384534

11. Raza M, Good Medical Record Keeping, International Journal of Collaborative Research on Internal Medicine \& Public Health, Volume 4.Issue 5, 2012Pages 535-543. http://internalmedicine.imedpub.com/

12. Sadvoski A, Simple NLP Search in your Application-step by step guide, http://www.tech.evojam.com/jun25,2017

13. Sinha P, Gaur S, Bendale P, Mantri M, Dande A (2012) Book Title Electronic Health Record, Standards, Coding Systems, Frameworks, and Infrastructures, IEEE Press ISBN 978-1-11828134-5

14. Srivastava, S. (2016). "Adoption of Electronic Health Records: A roadmap for India", Healthcare Informatics Research, 22(4), 261269 https://doi.org/10.4258/hir.2016.22.4.261

15. Tsung-Ting Kuo, Hyeon-Eui Kim, LucilaOhnoMachado, Blockchain distributed ledger technologies for biomedical and health care applications, Journal of the American Medical Informatics Association, Volume 24, Issue 6, November 2017, Pages 1211-1220, https://doi.org/10.1093/jamia/ocx068

16. Tejpal K, Blockchain: An Answer to Public EHR System, a Blog on https://www.investindia.gov.in/team-indiablogs/blockchain-answer-public-electronichealth-record-ehr-system (Last Accessed November 30, 2019) 


\begin{tabular}{|c|c|c|c|}
\hline 1 & Singapore & $\begin{array}{l}\text { National } \\
\text { Electronic } \\
\text { Health } \\
\text { Record } \\
\text { System } \\
\text { termed as } \\
\text { NEHR }\end{array}$ & 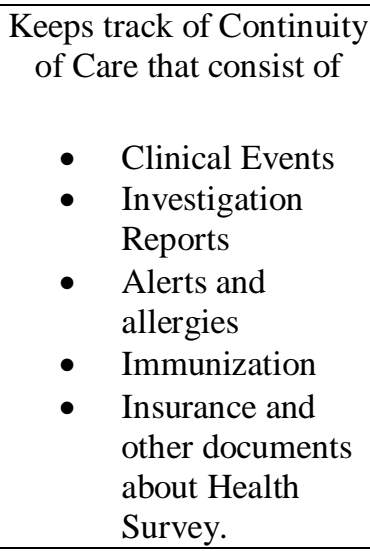 \\
\hline 2 & Norway & $\begin{array}{l}\text { PHC and EPR } \\
\text { Personal } \\
\text { Health Care } \\
\text { (PHC) Record } \\
\text { is for } \\
\text { Hospitals and } \\
\text { Early Primary } \\
\text { Record(EPR) } \\
\text { is for general } \\
\text { Practitioners } \\
\text { (GP) }\end{array}$ & $\begin{array}{l}\text { Extension of Meaningful } \\
\text { use of Health Record. }\end{array}$ \\
\hline 3 & Sweden & NPO & $\begin{array}{c}\text { Nationwide EHR } \\
\text { Framework that promotes } \\
\text { One Person One Record. }\end{array}$ \\
\hline
\end{tabular}

Figure 1 Typical HTML Page to record Patient Information One Person One Record.

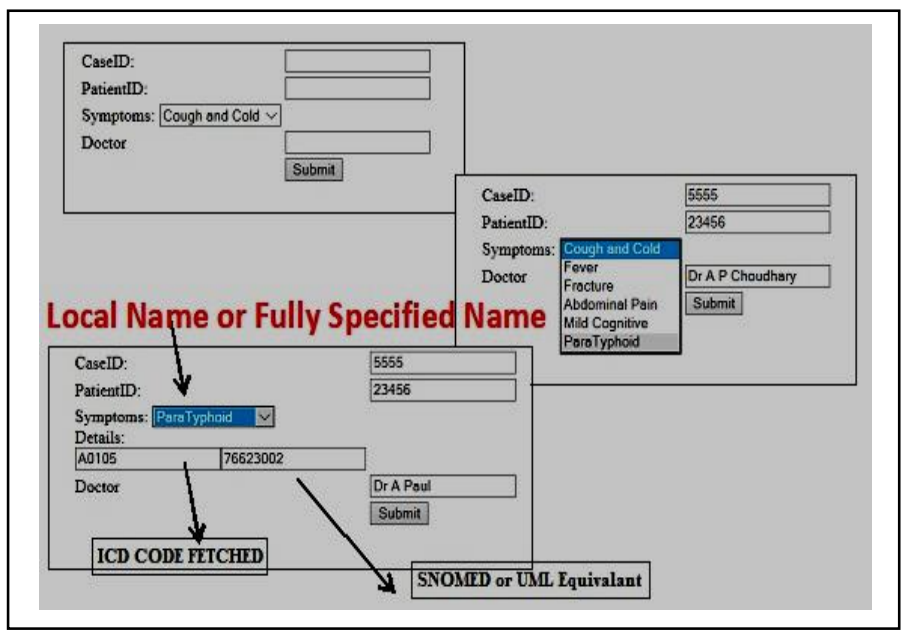

Figure 2 Using Mapping of standards using simple web techniques

Table 3: Select Health Care systems that can be used as reference to provide healthcare system in India.

\begin{tabular}{|l|l|l|l|}
$\mathrm{Sr}$ & Country & EHR System & Salient features \\
\hline
\end{tabular}

